九州大学学術情報リポジトリ

Kyushu University Institutional Repository

\title{
Synthesis and Insecticidal Activity of Bicyclic Phosphorothionates and Related Monocyclic Phosphorothionates
}

Wu, Shao-Yong

Laboratory of Pesticide Chemistry, Faculty of Agriculture, Kyushu University

Hirashima, Akinori

Laboratory of Pesticide Chemistry, Faculty of Agriculture, Kyushu University

Takeya, Ryuko

Laboratory of Pesticide Chemistry, Faculty of Agriculture, Kyushu University

Eto, Morifusa

Laboratory of Pesticide Chemistry, Faculty of Agriculture, Kyushu University

https://doi.org/10.5109/23939

出版情報 : 九州大学大学院農学研究院紀要. 33 (3/4)，pp. 275-285，1989-03. Kyushu University バージョン：

権利関係： 


\title{
Synthesis and Insecticidal Activity of Bicyclic Phosphorothionates and Related Monocyclic Phosphorothionates
}

\author{
Shao-Yong Wu*, Akinori Hirashima, Ryuko Takeya \\ and Morifusa Eto \\ Laboratory of Pesticide Chemistry, Faculty of Agriculture \\ Kyushu University 46-02, Fukuoka 812, Japan. \\ (Received November 11, 1988)
}

\begin{abstract}
Some thiono-type bicyclic phosphorus esters (BPs) were synthesized and bioassayed for insecticidal activity against susceptible (SRS) houseflies. n-Propyl was most favorable as a substituent at position 4 for insecticidal activity. The introduction of substituents at position 3 did not improve the activity. Some functionized monocyclic phosphorothionates (MPs) were also synthesized as proBP candidates. Although some MPs were cyclized to the corresponding BPs by oxidation or by action of a base, they did not show insecticidal activity. Some other MPs with a chloro-functionized 5-substituent had an insecticidal activity but their cyclization to the corresponding BPs was not yet proved.
\end{abstract}

\section{INTRODUCTION}

The structural variation of 2, 6, 7-trioxa-1-phosphabicyclo[2.2.2] octane or socalled bicyclic phosphorus ester (BP) is very limited and its synthetic modification has been mainly carried out on the 4-substituent as $\mathrm{RC}\left(\mathrm{CH}_{2} \mathrm{O}\right)_{3} \mathrm{P}=\mathrm{X}$ (Ozoe and Eto, 1982). Oxo-type BPs with a branched hydrophobic alkyl group at position 4 are highly toxic to mammals (Bellet and Casida, 1973 ; Eto et al., 1976) and potent noncompetitive $\gamma$ aminobutyric acid (GABA) antagonists (Bowery et al., 1976 ; Korenaga et al., 1977). Although these compounds have only weak insecticidal activity when topically applied to insects, considerably high insecticidal activity has been observed by injecting or by topically applying them with piperonyl butoxide (PB) (Ozoe et al., 1983, 198613). Introduction of an alkyl group at position 3 appears to increase selective toxicity to insects (Ozoe et al., 1983).

Propesticide concept is often useful for the structure modification of biologically active compounds in order to reduce mammalian toxicity and to increase pesticidal activity (Fukuto, 1984). 5-Substituted 1, 3, 2-dioxaphosphorinanes of the type $R_{1}$ $\left(\mathrm{HOCH}_{2}\right) \mathrm{C}\left(\mathrm{CH}_{2} \mathrm{O}\right)_{2} \mathrm{P}(\mathrm{O}) \mathrm{R}_{2}$ are potential proBPs by virtue of their cyclization ability and expected to be as candidates for propesticides (Toia and Casida, 1985, 1987).

Thiono-type BPs were not studied yet in detail as possible insecticides, though 4t-butylbicyclophosphorothionate (TBPS) is known as a specific ligand for the GABA receptor-ionophore complex (Squires et al., 1983) and a relatively high insecticidal activity was found in its $4-n$-propyl derivative (Ozoe et al., 1986a). This paper deals with the relationships between the structure and insecticidal activity of thiono-type BPs and monocyclic phosphorothionates (MPs) prepared as proBP candidates. Effect

\footnotetext{
*Present address : Institute of Applied Chemistry, Beijing Agricultural University, Beijing, China
} 
of substitution at the position 3 of BPs on insecticidal activity by topical application was also investigated.

\section{MATERIALS AND METHODS}

Synthesis of triols

Triols were synthesized by the following exemplified reactions.

2-Cyclohexyl-2-hydroxymethyl-1,3 -propanediol

\section{Cyclohexylacetaldehyde}

To pyridinium chlorochromate $(32.3 \mathrm{~g})$ suspended in $200 \mathrm{ml}$ of dichloromethane, cyclohexylethanol $(12.8 \mathrm{~g})$ in $20 \mathrm{ml}$ of dichloromethane was added. After stirred for $1.5 \mathrm{hr}, 200 \mathrm{ml}$ of dry ether was added and the supernatant was decanted from the black gum. The insoluble residue was washed with dry ether and the combined organic solution was passed through a short silica gel column, and then concentrated. Distillation gave the product in $65 \%$ yield, bp $74-75^{\circ} \mathrm{C}(18 \mathrm{mmHg}) .{ }^{1} \mathrm{H}-\mathrm{NMR}$ : $0.7-2.0$ $\left(11 \mathrm{H}, \mathrm{m}, \mathrm{C}_{6} \mathrm{H}_{11}\right), 2.2\left(2 \mathrm{H}, \mathrm{m}, \mathrm{CH}_{2} \mathrm{CO}\right), 10.4(1 \mathrm{H}, \mathrm{CHO})$.

\section{2-Cyclohexyl-2-hydroxymethyl-1,3-propanediol}

Cyclohexylacetaldehyde was transformed into 2-cyclohexyl-2-hydroxymethyl1, 3-propanediol by utilizing the Tollens condensation (Ozoe and Eto, 1982). Yield 16\%, bp $156-159^{\circ} \mathrm{C}(0.06 \mathrm{mmHg}), \mathrm{mp} 85^{\circ} \mathrm{C}$.

\section{2-Propyl-2-hydroxymethyl-1,3-butanediol}

\section{Diethyl Acetylpropylmalonate}

To sodium hydride $(0.1 \mathrm{~mol})$ in $75 \mathrm{ml}$ of dry ether, diethyl propylmalonate $(0.1 \mathrm{~mol})$ in $75 \mathrm{ml}$ of the same solvent was added dropwise and the mixture was refluxed for 3 $\mathrm{hr}$. To the reaction mixture, $100 \mathrm{ml}$ of a dry ether solution of acetyl chloride $(0.1 \mathrm{~mol})$ was added. After refluxed for $15 \mathrm{hr}$, the mixture was worked up in a usual way. Distillation gave the product in $74 \%$ yield, bp $77^{\circ} \mathrm{C}(0.1 \mathrm{mmHg})$. 'H-NMR : 0.94 [3H, $\left.\mathrm{t}, \mathrm{J}=7 \mathrm{~Hz}, \mathrm{CH}_{3}(\mathrm{Pr})\right], 1.30\left(2 \mathrm{H}, \mathrm{m}, \mathrm{MeCH}_{2} \mathrm{C}\right), 1.29\left[6 \mathrm{H}, \mathrm{t}, \mathrm{J}=7 \mathrm{~Hz},\left(\mathrm{COOCCH}_{3}\right)_{2}\right], 2.04$ $\left(2 \mathrm{H}, \mathrm{m}, \mathrm{EtCH}_{2}\right), 2.32\left(3 \mathrm{H}, \mathrm{s}, \mathrm{CH}_{3} \mathrm{CO}\right), 4.23\left[4 \mathrm{H}, \mathrm{q}, J=7 \mathrm{~Hz},\left(\mathrm{MeCH}_{2} \mathrm{O}\right)_{2}\right]$.

\section{2-Propyl-2-hydroxymethyl-1,3-butanediol}

Diethyl acetylpropylmalonate $(0.037 \mathrm{~mol})$ in $60 \mathrm{ml}$ of dry ether was added dropwise to a suspension of $\mathrm{LiAlH}_{4}(0.126 \mathrm{~mol})$ in $350 \mathrm{ml}$ of dry ether at $0^{\circ} \mathrm{C}$. After stirring at room temperature for 3 days, an aqueous potassium hydroxide solution (19 $\mathrm{g}$ in $50 \mathrm{ml}$ ) was carefully added to the reaction mixture, followed by addition of a solution containing $\mathrm{K}_{2} \mathrm{HPO}_{4}(14 \mathrm{~g})$ and $\mathrm{KH}_{2} \mathrm{PO}_{4}(11 \mathrm{~g})$ in $\mathrm{H}_{2} \mathrm{O}(50 \mathrm{ml})$. After evaporation of ether, the aqueous solution was neutralized with acetic acid and concentrated. The residue was extracted with acetone and evaporation of the solvent gave crystals which were used for next synthesis without further purification.

\section{Synthesis of BPs}

BPs were synthesized in a similar manner as described by Ozoe and Eto (1982). 4-Ethyl-2, 6, 7-trioxa-1-phosphabicyclo [2.2.2] octane 1-Oxide (BP-l)

${ }^{1} \mathrm{H}-\mathrm{NMR}: 0.90\left(3 \mathrm{H}, \mathrm{t}, J=7 \mathrm{~Hz}, \mathrm{CH}_{3} \mathrm{C}\right), 1.36\left(2 \mathrm{H}, \mathrm{q}, J=7 \mathrm{~Hz}, \mathrm{MeCH}_{2}\right), 4.49[6 \mathrm{H}, \mathrm{d}$, $\left.\mathrm{J}=6 \mathrm{~Hz},\left(\mathrm{CH}_{2} \mathrm{O}\right)_{3}\right] .{ }^{31} \mathrm{P}-\mathrm{NMR}:-7.4$. MS : $178\left(\mathrm{M}^{+}, 10\right), 150(32), 68$ (100).

4-Ethyl-2, 6, 7-trioxa-1-phosphabicyclo [2.2.2] octane 1-Sulfide (BP-2)

${ }^{1} \mathrm{H}-\mathrm{NMR}: 0.88(3 \mathrm{H}, \mathrm{t}, \mathrm{J}=7 \mathrm{~Hz}, \mathrm{CH}),, 1.32\left(2 \mathrm{H}, \mathrm{q}, \mathrm{J}=7 \mathrm{~Hz}, \mathrm{MeCH}_{2}\right), 4.46[6 \mathrm{H}, \mathrm{d}$, 
$\left.J=6 \mathrm{~Hz},\left(\mathrm{CH}_{2} \mathrm{O}\right)_{3}\right] .{ }^{31} \mathrm{P}-\mathrm{NMR}:$ 52.2. MS : $194\left(\mathrm{M}^{+}, 100\right)$.

4-Phenyl-2, 6, 7-trioxa-1-phosphabicyclo[2.2.2] octane 1-Sulfide (BP-3)

'H-NMR : $4.82\left[6 \mathrm{H}, \mathrm{d}, J=6 \mathrm{~Hz},\left(\mathrm{CH}_{2} \mathrm{O}\right)_{3}\right], 7.0-7.5(5 \mathrm{H}, \mathrm{m}, \mathrm{Ph})$.

4-Cyclohexyl-2, 6, 7-trioxa-1-phosphabicyclo[2.2.2] octane I-Sulfide (BP-4)

'H-NMR : 0.8-1.9 (11H, $\left.\mathrm{C}_{6} \mathrm{H}_{11}\right), 4.49\left[6 \mathrm{H}, \mathrm{d}, J=6 \mathrm{~Hz},\left(\mathrm{CH}_{2} \mathrm{O}\right)_{3}\right]$.

4-Propyl-2, 6, 7-trioxa-1-phosphabicyclo [2.2.2] octane 1-Sulfide (BP-5)

${ }^{1} \mathrm{H}-\mathrm{NMR}: 0.92(3 \mathrm{H}, \mathrm{t}, J=7 \mathrm{~Hz}, \mathrm{CH}),, 1.23\left(4 \mathrm{H}, \mathrm{m}, \mathrm{MeCH}_{2} \mathrm{CH}_{2}\right), 4.46[6 \mathrm{H}, \mathrm{d}, J=$ $\left.6 \mathrm{~Hz},\left(\mathrm{CH}_{2} \mathrm{O}\right)_{3}\right]$. MS : $208(\mathrm{M}+, 81), 83$ (47), 79 (57), 55 (100).

3-Methyl-4-propyl-2, 6, 7-trioxa-1-phosphabicyclo[2.2.2] octane 1-Sulfide (BP-6)

'H-NMR : $0.91\left[3 \mathrm{H}, \mathrm{t}, J=6 \mathrm{~Hz}, \mathrm{CH}_{3}(\mathrm{Pr})\right], 1.16\left(4 \mathrm{H}, \mathrm{m}, \mathrm{MeCH}_{2} \mathrm{CH}_{2}\right), 1.50(3 \mathrm{H}, 2 \mathrm{~d}$, $\left.J=6 \mathrm{~Hz}, \mathrm{OCCH}_{3}\right), 4.45\left[4 \mathrm{H}, \mathrm{d}, J=7 \mathrm{~Hz},\left(\mathrm{CH}_{2} \mathrm{O}\right)_{2}\right], 4.72(1 \mathrm{H}, \mathrm{m}, \mathrm{MeCH}) . \mathrm{MS}: 222\left(\mathrm{M}^{+}\right.$, 100).

3-Ethyl-4-propyl-2, 6, 7-trioxa-1-phosphabicyclo [2.2.2] octane 1-Sulfide (BP-7)

'H-NMR : $0.91\left[3 \mathrm{H}, \mathrm{t}, J=6 \mathrm{~Hz}, \mathrm{CH}_{3}(\mathrm{Pr})\right], 1.16\left[3 \mathrm{H}, \mathrm{t}, J=7 \mathrm{~Hz}, \mathrm{CH}_{3}(\mathrm{Et})\right], 1.2(4 \mathrm{H}$, $\left.\mathrm{m}, \mathrm{MeCH}_{2} \mathrm{CH}_{2}\right), 1.78\left(2 \mathrm{H}, \mathrm{m}, \mathrm{MeCH}_{2} \mathrm{CO}\right), 4.4(1 \mathrm{H}, \mathrm{m}, \mathrm{EtCH}), 4.45\left[4 \mathrm{H}, \mathrm{d}, J=7 \mathrm{~Hz},\left(\mathrm{CH}_{2}-\right.\right.$ O),], MS : $236\left(\mathrm{M}^{+}, 100\right)$.

3-Isopropyl-4-propyl-2, 6, 7-trioxa-1-phosphabicyclo [2.2.2] octane 1-Sulfide (BP-8)

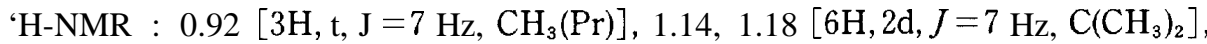
$1.25\left(4 \mathrm{H}, \mathrm{m}, \mathrm{MeCH}_{2} \mathrm{CH}_{2}\right), 2.13\left(1 \mathrm{H}, \mathrm{m}, \mathrm{Me}_{2} \mathrm{CH}\right), 4.40\left[4 \mathrm{H}, \mathrm{d}, J=7 \mathrm{~Hz},\left(\mathrm{CH}_{2} \mathrm{O}\right)_{2}\right], 4.5(1 \mathrm{H}$, m, CHO). MS : $250\left(\mathrm{M}^{+}, 100\right)$.

3-Phenyl-4-propyl-2, 6, 7-trioxa-1-phosphabicyclo [2.2.2] octane 1-Sulfide (BP-9)

'H-NMR : 0.6-1.4 (7H, m, $\left.\mathrm{CH}_{3} \mathrm{CH}_{2} \mathrm{CH}_{2}\right), 4.30,4.58\left[4 \mathrm{H}, 2 \mathrm{~d}, J=7 \mathrm{~Hz},\left(\mathrm{CH}_{2} \mathrm{O}\right)_{2}\right]$, $5.59(1 \mathrm{H}, \mathrm{d}, J=7 \mathrm{~Hz}, \mathrm{CHO}), 7.38(5 \mathrm{H}, \mathrm{s}, \mathrm{Ph}) . \mathrm{MS}: 284\left(\mathrm{M}^{+}, 59\right), 91$ (100).

3-Benzyl-4-propyl-2, 6, 7-trioxa-1-phosphabicyclo[2.2.2] octane l-sulfide (BP-IO)

${ }^{1} \mathrm{H}-\mathrm{NMR}: 1.04\left(3 \mathrm{H}, \mathrm{t}, J=7 \mathrm{~Hz}, \mathrm{CH}_{3}\right), 1.2-1.8\left(4 \mathrm{H}, \mathrm{m}, \mathrm{MeCH}_{2} \mathrm{CH}_{2}\right), 2.3-2.9(2 \mathrm{H}, \mathrm{m}$, $\left.\mathrm{PhCH}_{2}\right), 4.0-4.8\left[4 \mathrm{H}, \mathrm{m},\left(\mathrm{CH}_{2} \mathrm{O}\right)_{2}\right], 4.80(1 \mathrm{H}, \mathrm{m}, \mathrm{CHO}) . \mathrm{MS}: 298\left(\mathrm{M}^{+}, 3\right), 243(22), 92(75)$, $58(100)$.

\section{Synthesis of monocyclic phosphorothionates (MPs)}

Monocyclic phosphorothionates (MPs) were synthesized by the reaction of a trio1 and a thiophosphoryl dichloride. In some cases, the methylol group at position 5 was protected with one of acetyl, mesyl or tosyl group by treating with the corresponding chloride. Others were converted to halides by using thionyl halide.

5-Chloromethyl-5-ethyl-2-methoxy-1, 3, 2-dioxaphosphorinane 2-Sulfide (MP-11) (MP-3)

1. 5-Ethyl-5-hydroxymethyl-2-methoxy-1,3,2-dioxaphosphorinane 2-Sulfide

To the mixture of methyl phosphorodichloridothionate $(0.01 \mathrm{~mol})$ and 2-ethyl-2hydroxymethyl-1,3-propanediol $(0.01 \mathrm{~mol})$ in $5 \mathrm{ml}$ of $\mathrm{H}_{2} \mathrm{O}, \mathrm{NaOH}(0.02 \mathrm{~mol})$ was added at $0^{\circ} \mathrm{C}$. After stirred at $0^{\circ} \mathrm{C}$ for $1 \mathrm{hr}$ and at room temperature for $1 \mathrm{hr}$, the mixture was neutralized and extracted with ether. The product as a diastereomeric mixture was obtained after purification through column chromatography using a mixture of benzene and ether as a gradient elution system. Yield 33\%. 'H-NMR : $0.92(3 \mathrm{H}, \mathrm{t}, J=$ $7 \mathrm{~Hz}, \mathrm{CCH},), 1.30,1.64\left(2 \mathrm{H}, 2 \mathrm{q}, J=7 \mathrm{~Hz}, \mathrm{MeCH}_{2}\right), 2.4,2.7(1 \mathrm{H}, \mathrm{OH}), 3.54,3.80(2 \mathrm{H}, 2 \mathrm{~s}$, $\left.\mathrm{CH}_{2} \mathrm{O}\right), 3.99,4.00\left(3 \mathrm{H}, 2 \mathrm{~d}, J=14 \mathrm{~Hz}, \mathrm{POCH}_{3}\right), 3.90-4.46\left[4 \mathrm{H}, \mathrm{m},\left(\mathrm{CH}_{2} \mathrm{O}\right)_{2}\right] . \mathrm{MS}: 226$ $\left(\mathrm{M}^{+}, 10\right), 129$ (100).

2. 5-Chloromethyl-5-ethyl-2-methoxy-1,3,2-dioxaphosphorinane 2-Sulfide (MP- 
11)

To a solution of 5-ethyl-5-hydroxymethyl-2-methoxy-1,3,2-dioxaphosphorinane Z-sulfide $(0.31 \mathrm{mmol})$ in hexamethyl phosphoramidate $(4 \mathrm{ml})$, thionyl chloride $(1.5 \mathrm{ml})$ was added slowly. After the mixture was stirred at room temperature for $12 \mathrm{hr}, \mathrm{H}_{2} \mathrm{O}$ $(5 \mathrm{ml})$ and ether $(30 \mathrm{ml})$ were added. The ether layer was washed with successive $5 \%$ $\mathrm{NaOH}$ and saturated $\mathrm{NaCl}$ aq., and dried over anhydrous $\mathrm{Na}_{2} \mathrm{SO}_{4}$. The product was purified by column chromatography using a mixture of benzene and ether as a gradient elution system. Yield 46\%. The cis- and trans-isomers were separated by a Lobar column using a mixture of hexane and ether as a gradient elution system. Cis-isomer, 'H-NMR : $0.89(3 \mathrm{H}, \mathrm{t}, \mathrm{J}=7 \mathrm{~Hz}, \mathrm{CCH}), 1.40\left(2 \mathrm{H}, \mathrm{q}, J=7 \mathrm{~Hz}, \mathrm{MeCH}_{2}\right), 3.81(3 \mathrm{H}, \mathrm{d}, J=$ $14 \mathrm{~Hz}, \mathrm{POCH},), 3.82\left(2 \mathrm{H}, \mathrm{s}, \mathrm{CH}_{2} \mathrm{Cl}\right), 4.1-4.3\left[4 \mathrm{H}, \mathrm{m},\left(\mathrm{CH}_{2} \mathrm{O}\right)_{2}\right] . \mathrm{MS}: 244\left(\mathrm{M}^{+}, 52\right), 129$ (100) ; trans-isomer, 'H-NMR : $0.92(3 \mathrm{H}, \mathrm{t}, J=7 \mathrm{~Hz}, \mathrm{CCH}),, 1.63\left(2 \mathrm{H}, \mathrm{q}, \mathrm{MeCH}_{2}\right), 3.57$ $\left(2 \mathrm{H}, \mathrm{s}, \mathrm{CH}_{2} \mathrm{Cl}\right), 3.86(3 \mathrm{H}, \mathrm{d}, J=14 \mathrm{~Hz}, \mathrm{POCH}),, 4.26\left[4 \mathrm{H}, \mathrm{d}, J=14 \mathrm{~Hz},\left(\mathrm{CH}_{2} \mathrm{O}\right)_{2}\right] . \mathrm{MS}$ : $244(\mathrm{M}+, 30), 129(100)$.

The following dioxaphosphorinanes were prepared in a similar manner.

5-Ethyl-5-hydroxymethyl-2-p-nitrophenoxy-1, 3, 2-dioxaphosphorinane Z-Sulfide (MP- 1)

Cis-isomer, 'H-NMR : $0.92(3 \mathrm{H}, \mathrm{t}, J=7 \mathrm{~Hz} . \mathrm{CCH}), 1.41\left(2 \mathrm{H}, \mathrm{q}, J=7 \mathrm{~Hz}, \mathrm{MeCH}_{2}\right)$, $1.9(1 \mathrm{H}, \mathrm{OH}), 3.94\left(2 \mathrm{H}, \mathrm{s}, \mathrm{CH}_{2} \mathrm{O}\right), 4.1-4.5\left[4 \mathrm{H}, \mathrm{m},\left(\mathrm{CH}_{2} \mathrm{O}\right)_{2}\right], 6.34,7.23(4 \mathrm{H}, \mathrm{m}$, aromatic). ${ }^{31} \mathrm{P}-\mathrm{NMR}$ : 53.5. MS : 333 (M+, 10), 236 (96), 179 (100). Trans-isomer, 'H-NMR : 0.99 $\left(3 \mathrm{H}, \mathrm{t}, J=7 \mathrm{~Hz}, \mathrm{CCH}_{3}\right), 1.77\left(2 \mathrm{H}, \mathrm{q}, J=7 \mathrm{~Hz}, \mathrm{MeCH}_{2}\right), 3.56\left(2 \mathrm{H}, \mathrm{s}, \mathrm{CH}_{2} \mathrm{O}\right), 4.1-4.7$ [ $4 \mathrm{H}$, $\left.\mathrm{m},\left(\mathrm{CH}_{2} \mathrm{O}\right)_{2}\right], 6.39,7.24\left(4 \mathrm{H}, \mathrm{m}\right.$, aromatic). ${ }^{31} \mathrm{P}-\mathrm{NMR}: 54.7$.

5-Acetoxymethyl-5-ethyl-2-p-nitrophenoxy-1，3，2-dioxaphosphorinane 2-Sulfide (MP-2)

'H-NMR : 0.90, $1.00\left(3 \mathrm{H}, 2 \mathrm{t}, J=7 \mathrm{~Hz}, \mathrm{CCH}_{3}\right), 1.3,1.7(1 \mathrm{H}, 2 \mathrm{~s}, \mathrm{OH}), 1.38,1.78(2 \mathrm{H}$, $\left.2 \mathrm{q}, J=7 \mathrm{~Hz}, \mathrm{MeCH}_{2}\right), 2.08,2.12(3 \mathrm{H}, 2 \mathrm{~s}, \mathrm{OCOCH}), 3.97,,4.36\left(2 \mathrm{H}, 2 \mathrm{~s}, \mathrm{CH}_{2} \mathrm{O}\right), 4.1-4.5$ $\left[4 \mathrm{H}, \mathrm{m},\left(\mathrm{CH}_{2} \mathrm{O}\right)_{2}\right], 6.32,7.24\left(4 \mathrm{H}, \mathrm{m}\right.$, aromatic). MS : $375\left(\mathrm{M}^{+}, 15\right), 236(87), 179(100)$. 5-Acetoxymethyl-5-ethyl-2-methoxy-1,3, 2-dioxaphosphorinane 2-Sulfide (MP-4)

${ }^{1} \mathrm{H}-\mathrm{NMR}: 0.88,0.92\left(3 \mathrm{H}, 2 \mathrm{t}, J=7 \mathrm{~Hz}, \mathrm{CCH}_{3}\right), 1.36,1.62\left(2 \mathrm{H}, 2 \mathrm{q}, J=7 \mathrm{~Hz}, \mathrm{MeCH}_{2}\right)$, $2.08(3 \mathrm{H}, \mathrm{s}, \mathrm{OCOCH}), 3.80,,3.84\left(3 \mathrm{H}, 2 \mathrm{~d}, J=14 \mathrm{~Hz}, \mathrm{POCH}_{3}\right), 3.9-4.3\left[6 \mathrm{H}, \mathrm{m},\left(\mathrm{CH}_{2} \mathrm{O}\right)_{2}\right.$, $\left.\mathrm{CH}_{2} \mathrm{OCO}\right]$. MS : $268\left(\mathrm{M}^{+}, 94\right), 171(70), 129$ (100).

5-Ethyl-5-hydroxymethyl-2-methylthio-1,3, 2-dioxaphosphorinane 2-Sulfide (MP-5)

Cis-isomer, 'H-NMR : $0.93\left(3 \mathrm{H}, \mathrm{t}, J=7 \mathrm{~Hz}, \mathrm{CCH}_{3}\right), 1.65\left(2 \mathrm{H}, \mathrm{q}, J=7 \mathrm{~Hz}, \mathrm{MeCH}_{2}\right)$, $1.83(1 \mathrm{H}, \mathrm{OH}), 2.42\left(3 \mathrm{H}, \mathrm{d}, J=17 \mathrm{~Hz}, \mathrm{PSCH}_{3}\right), 3.59\left(2 \mathrm{H}, \mathrm{s}, \mathrm{CH}_{2} \mathrm{O}\right), 4.0-4.4\left[4 \mathrm{H}, \mathrm{m},\left(\mathrm{CH}_{2}{ }^{-}\right.\right.$ $\mathrm{O}_{2}$ ]. Trans-isomer, 'H-NMR : $0.90(3 \mathrm{H}, \mathrm{t}, J=7 \mathrm{~Hz}, \mathrm{CCH}), 1.38(2 \mathrm{H}, \mathrm{q}, J=7 \mathrm{~Hz}$, $\left.\mathrm{MeCH}_{2}\right), 1.92(1 \mathrm{H}, \mathrm{OH}), 2.41\left(3 \mathrm{H}, \mathrm{d}, J=17 \mathrm{~Hz}, \mathrm{PSCH}_{3}\right), 3.85\left(2 \mathrm{H}, \mathrm{s}, \mathrm{CH}_{2} \mathrm{O}\right), 4.0-4.4[4 \mathrm{H}$, $\left.\mathrm{m},\left(\mathrm{CH}_{2} \mathrm{O}\right)_{2}\right]$. MS : $242(\mathrm{M}+, 100), 195(90)$.

5-Acetoxymethyl-5-ethyl-2-methylthio-1,3, 2-dioxaphosphorinane 2-Sulfide (MP-6)

Cis-isomer, 'H-NMR : $0.92\left(3 \mathrm{H}, \mathrm{t}, J=7 \mathrm{~Hz}, \mathrm{CCH}_{3}\right), 1.64\left(2 \mathrm{H}, \mathrm{q}, J=7 \mathrm{~Hz}, \mathrm{MeCH}_{2}\right)$, $2.10(3 \mathrm{H}, \mathrm{s}, \mathrm{OOCCH}),, 2.43\left(3 \mathrm{H}, \mathrm{d}, J=17 \mathrm{~Hz}, \mathrm{PSCH}_{3}\right), 4.0-4.4\left[6 \mathrm{H}, \mathrm{m},\left(\mathrm{CH}_{2} \mathrm{O}\right)_{3}\right] . \mathrm{MS}$ : $284\left(\mathrm{M}^{+}, 64\right), 237(27), 145(100)$. Trans-isomer, 'H-NMR : $0.88\left(3 \mathrm{H}, \mathrm{t}, J=7 \mathrm{~Hz}, \mathrm{CCH}_{3}\right)$, $1.37\left(2 \mathrm{H}, \mathrm{q}, J=7 \mathrm{~Hz}, \mathrm{MeCH}_{2}\right), 2.09\left(3 \mathrm{H}, \mathrm{s}, \mathrm{OOCCH}_{3}\right), 2.41\left(3 \mathrm{H}, \mathrm{d}, J=17 \mathrm{~Hz}, \mathrm{PSCH}_{3}\right)$, 4.0-4.4 [6H, m, $\left.\left(\mathrm{CH}_{2} \mathrm{O}\right)_{3}\right]$. MS : $284\left(\mathrm{M}^{+}, 100\right), 237(60), 145$ (61).

5-Ethyl-2-ethylthio-5-hydroxymethyl-1,3, 2-dioxaphosphorinane 2-Sulfide (MP-7)

Cis-isomer, 'H-NMR : $0.93\left(3 \mathrm{H}, \mathrm{t}, J=7 \mathrm{~Hz}, \mathrm{CCCH}_{3}\right), 1.39\left(3 \mathrm{H}, \mathrm{t}, J=7 \mathrm{~Hz}, \mathrm{SCCH}_{3}\right)$, $1.63\left(2 \mathrm{H}, \mathrm{q}, J=7 \mathrm{~Hz}, \mathrm{MeCH}_{2} \mathrm{C}\right), 1.76(1 \mathrm{H}, \mathrm{OH}), 2.94,3.12\left(2 \mathrm{H}, 2 \mathrm{q}, J=7 \mathrm{~Hz}, \mathrm{MeCH}_{2} \mathrm{~S}\right)$, 
$3.58\left(2 \mathrm{H}, \mathrm{s}, \mathrm{CH}_{2} \mathrm{O}\right), 4.0-4.5\left(4 \mathrm{H}, \mathrm{m},\left(\mathrm{CH}_{2} \mathrm{O}\right)_{2}\right] .{ }^{31} \mathrm{P}-\mathrm{NMR}:$ 95.98. Trans-isomer, ${ }^{1} \mathrm{H}-$ NMR : 0.89 [3H, t, J =7 Hz, CCCH,), 1.38 (3H, t, J = 7 Hz, SCCH,), 1.2-1.5 (2H, q, J = $\left.7 \mathrm{~Hz}, \mathrm{MeCH}_{2} \mathrm{C}\right), 2.38(1 \mathrm{H}, J=5 \mathrm{~Hz}, \mathrm{OH}), 2.92,3.08\left(2 \mathrm{H}, 2 \mathrm{q}, \mathrm{J}=7 \mathrm{~Hz}, \mathrm{MeCH}_{2} \mathrm{~S}\right), 3.81(2 \mathrm{H}$, $\left.\mathrm{d}, \mathrm{J}=5 \mathrm{~Hz}, \mathrm{CH}_{2} \mathrm{O}\right), 3.9-4.4\left[4 \mathrm{H}, \mathrm{m},\left(\mathrm{CH}_{2} \mathrm{O}\right)_{2}\right] .{ }^{31} \mathrm{P}-\mathrm{NMR}: 90.57$.

5-Ethyl-2-methoxy-5-mesyloxymethyl-1,3, 2-dioxaphosphorinane 2-Sulfide (MP-8)

'H-NMR : 0.91, $0.95\left(3 \mathrm{H}, 2 \mathrm{t}, J=7 \mathrm{~Hz}, \mathrm{CCH}_{3}\right), 1.40,1.60\left(2 \mathrm{H}, 2 \mathrm{q}, J=7 \mathrm{~Hz}, \mathrm{MeCH}_{2}\right)$, $3.41\left(3 \mathrm{H}, \mathrm{s}, \mathrm{O}_{3} \mathrm{SCH}_{3}\right), 3.80,3.86\left(3 \mathrm{H}, 2 \mathrm{~d}, J=14 \mathrm{~Hz}, \mathrm{POCH}_{3}\right), 3.91,4.23\left(2 \mathrm{H}, 2 \mathrm{~s}, \mathrm{MeSO}_{3}{ }^{-}\right.$ $\mathrm{CH}$,$\left.) , 4.0-4.5 [4H, m, \left(\mathrm{CH}_{2} \mathrm{O}\right)_{2}\right]$. MS : $304\left(\mathrm{M}^{+}, 48\right), 225(100)$.

5-Ethyl-2-methoxy-5-tosyloxymethyl-1,3, 2-dioxaphosphorinane 2-Sulfide (MP-9)

'H-NMR: $0.81\left(3 \mathrm{H}, \mathrm{t}, J=7 \mathrm{~Hz}, \mathrm{CCH}_{3}\right), 1.34,1.53\left(2 \mathrm{H}, 2 \mathrm{q}, J=7 \mathrm{~Hz}, \mathrm{MeCH}_{2}\right), 2.44$ $\left(3 \mathrm{H}, \mathrm{s}, \mathrm{PhCH}_{3}\right), 3.74,3.76\left(3 \mathrm{H}, 2 \mathrm{~d}, J=14 \mathrm{~Hz}, \mathrm{POCH}_{3}\right), 3.9-4.2\left[6 \mathrm{H}, \mathrm{m},\left(\mathrm{CH}_{2} \mathrm{O}\right)_{3}\right], 7.2-$ $7.8\left(4 \mathrm{H}, \mathrm{m}\right.$, aromatic). MS : $380\left(\mathrm{M}^{+}, 26\right), 225(100)$.

5-Bromomethyl-5-ethyl-2-methoxy-1, 3, 2-dioxaphosphorinane 2-Sulfide (MP-10)

'H-NMR: $0.92\left(3 \mathrm{H}, \mathrm{t}, J=7 \mathrm{~Hz}, \mathrm{CCH}_{3}\right), 1.2-1.8\left(2 \mathrm{H}, \mathrm{q}, J=7 \mathrm{~Hz}, \mathrm{MeCH}_{2}\right), 3.42,3.70$ $\left(2 \mathrm{H}, 2 \mathrm{~s}, \mathrm{CH}_{2} \mathrm{Br}\right), 3.81,3.85\left(3 \mathrm{H}, 2 \mathrm{~d}, J=14 \mathrm{~Hz}, \mathrm{POCH}_{3}\right), 4.1-4.4\left[4 \mathrm{H}, \mathrm{m},\left(\mathrm{CH}_{2} \mathrm{O}\right)_{2}\right] . \mathrm{MS}$ : $290(M++2,100), 288(\mathrm{M}+, 92)$.

2-Chloro-5-hydroxymethyl-5-ethyl-1, 3, 2-dioxaphosphorinane 2-Sulfide (MP-12)

G-isomer, 'H-NMR : $0.97\left(3 \mathrm{H}, \mathrm{t}, J=7 \mathrm{~Hz}, \mathrm{CCH}_{3}\right), 1.78\left(2 \mathrm{H}, \mathrm{q}, J=7 \mathrm{~Hz}, \mathrm{MeCH}_{2}\right)$, $1.82(1 \mathrm{H}, \mathrm{s}, \mathrm{OH}), 3.48\left(2 \mathrm{H}, s, \mathrm{CH}_{2} \mathrm{O}\right), 3.9-4.7\left[4 \mathrm{H}, \mathrm{m},\left(\mathrm{CH}_{2} \mathrm{O}\right)_{2}\right] . \mathrm{MS}: 230(\mathrm{M}+, 47), 133$ (91), 98 (100).

2-Chloro-5-chloromethyl-5-ethyl-1, 3, 2-dioxaphosphorinane 2-Sulfide (MP-13)

Cis-isomer, 'H-NMR : $0.99\left(3 \mathrm{H}, \mathrm{t}, J=7 \mathrm{~Hz}, \mathrm{CCH}_{3}\right), 1.92\left(2 \mathrm{H}, \mathrm{q}, J=7 \mathrm{~Hz}, \mathrm{MeCH}_{2}\right)$, $3.37\left(2 \mathrm{H}, s, \mathrm{CH}_{2} \mathrm{Cl}\right), 3.9-4.6\left[4 \mathrm{H}, \mathrm{m},\left(\mathrm{CH}_{2} \mathrm{O}\right)_{2}\right] . \mathrm{MS}: 248\left(\mathrm{M}^{+}, 30\right), 116(82), 81(100)$; trans-isomer, 'H-NMR : $0.92\left(3 \mathrm{H}, \mathrm{t}, \mathrm{J}=7 \mathrm{~Hz}, \mathrm{CCH}_{3}\right), 1.43\left(2 \mathrm{H}, \mathrm{q}, \mathrm{J}=7 \mathrm{~Hz}, \mathrm{MeCH}_{2}\right)$, $3.82\left(2 \mathrm{H}, \mathrm{s}, \mathrm{CH}_{2} \mathrm{Cl}\right), 4.0-4.6\left[4 \mathrm{H}, \mathrm{m},\left(\mathrm{CH}_{2} \mathrm{O}\right)_{2}\right] . \mathrm{MS}: 248\left(\mathrm{M}^{+}, 42\right), 116(94), 81(100)$.

5-Chloromethyl-2-methoxy-5-propyl-1,3, 2-dioxaphosphorinane 2-Sulfide (MP-14)

'H-NMR : cis-isomer, $0.88\left(3 \mathrm{H}, \mathrm{t}, \mathrm{J}=7 \mathrm{~Hz}, \mathrm{CCH}_{3}\right), 1.28\left(4 \mathrm{H}, \mathrm{m}, \mathrm{MeCH}_{2} \mathrm{CH}_{2}\right), 3.77$ $\left(3 \mathrm{H}, \mathrm{d}, J=14 \mathrm{~Hz}, \mathrm{POCH}_{3}\right), 3.80\left(2 \mathrm{H}, \mathrm{s}, \mathrm{CH}_{2} \mathrm{Cl}\right) 4.0-4.3\left[4 \mathrm{H}, \mathrm{m},\left(\mathrm{CH}_{2} \mathrm{O}\right)_{2}\right]$; bans-isomer, $0.96\left(3 \mathrm{H}, \mathrm{t}, J=7 \mathrm{~Hz}, \mathrm{CCH}_{3}\right), 1.1-1.7\left(4 \mathrm{H}, \mathrm{m}, \mathrm{MeCH}_{2} \mathrm{CH}_{2}\right), 3.59\left(2 \mathrm{H}, \mathrm{s}, \mathrm{CH}_{2} \mathrm{Cl}\right), 3.85(3 \mathrm{H}$, $\left.\mathrm{d}, J=14 \mathrm{~Hz}, \mathrm{POCH}_{3}\right), 4.24\left[4 \mathrm{H}, \mathrm{d}, \mathrm{J}=14 \mathrm{~Hz},\left(\mathrm{CH}_{2} \mathrm{O}\right)_{2}\right]$.

5-(1-Chloroethyl)-2-methoxy-5-propyl-1,3,2-dioxaphosphorinane 2-Sulfide (MP15)

Cis-isomer, 'H-NMR : $0.92\left(3 \mathrm{H}, \mathrm{t}, J=7 \mathrm{~Hz}, \mathrm{CCH}_{3}\right), 1.1-1.5\left(4 \mathrm{H}, \mathrm{m}, \mathrm{MeCH}_{2} \mathrm{CH}_{2}\right)$, $1.61\left(3 \mathrm{H}, \mathrm{d}, J=7 \mathrm{~Hz}, \mathrm{CH}_{3} \mathrm{CCl}\right), 3.80\left(3 \mathrm{H}, \mathrm{d}, J=14 \mathrm{~Hz}, \mathrm{POCH}_{3}\right), 4.1-4.4\left[4 \mathrm{H}, \mathrm{m},\left(\mathrm{CH}_{2}-\right.\right.$ $\mathrm{O}_{2}$ ], $4.62(1 \mathrm{H}, \mathrm{q}, \mathrm{J}=7 \mathrm{~Hz}, \mathrm{MeCHCl}) . \mathrm{MS}: 272(\mathrm{M}+, 15), 195$ (23), 129 (100) ; transisomer, 'H-NMR : $0.98\left(3 \mathrm{H}, \mathrm{t}, J=7 \mathrm{~Hz}, \mathrm{CCCH}_{3}\right), 1.1-1.9\left(4 \mathrm{H}, \mathrm{m}, \mathrm{MeCH}_{2} \mathrm{CH}_{2}\right), 1.52(3 \mathrm{H}$, $\left.\mathrm{d}, J=7 \mathrm{~Hz}, \mathrm{CH}_{3} \mathrm{CCl}\right), 3.83\left(3 \mathrm{H}, \mathrm{d}, J=14 \mathrm{~Hz}, \mathrm{POCH}_{3}\right), 4.20(1 \mathrm{H}, \mathrm{q}, J=7 \mathrm{~Hz}, \mathrm{MeCHCl})$, 4.0-4.6 $\left[4 \mathrm{H}, \mathrm{m},\left(\mathrm{CH}_{2} \mathrm{O}\right)_{2}\right]$.

5-(1-Chloropropyl)-2-methoxy-5-propyl-1, 3, 2-dioxaphosphorinane 2-Sulfide (MP16)

${ }^{1} \mathrm{H}-\mathrm{NMR}: 0.94\left[3 \mathrm{H}, \mathrm{t}, J=7 \mathrm{~Hz}, \mathrm{CH}_{3}(\mathrm{Pr})\right], 1.14\left(3 \mathrm{H}, \mathrm{t}, J=7 \mathrm{~Hz}, \mathrm{CH}_{3} \mathrm{CCCl}\right), 1.1-$ $1.8\left(6 \mathrm{H}, \mathrm{m}, \mathrm{MeCH}_{2} \mathrm{CH}_{2}, \mathrm{MeCH}_{2} \mathrm{CCl}\right), 3.78,3.80\left(3 \mathrm{H}, 2 \mathrm{~d}, J=14 \mathrm{~Hz}, \mathrm{POCH}_{3}\right), 4.0-4.6[4 \mathrm{H}$, $\left.\mathrm{m},\left(\mathrm{CH}_{2} \mathrm{O}\right)_{2}\right], 4.28,4.32(1 \mathrm{H}, 2 \mathrm{t}, J=7 \mathrm{~Hz}, \mathrm{CHCl}) . \mathrm{MS}: 286\left(\mathrm{M}^{+}, 2\right), 129(75), 93(100)$. 5-Isobutenyl-2-methoxy-5-propyl-1, 3, 2-dioxaphosphorinane 2-Sulfide (MP-17)

'H-NMR : $0.94\left[3 \mathrm{H}, \mathrm{t}, J=7 \mathrm{~Hz}, \mathrm{CH}_{3}(\mathrm{Pr})\right], 1.1-1.7\left(4 \mathrm{H}, \mathrm{m}, \mathrm{MeCH}_{2} \mathrm{CH}_{2}\right), 1.74[6 \mathrm{H}$, $\left.\mathrm{C}\left(\mathrm{CH}_{3}\right)_{2}\right], 3.76,3.80\left(3 \mathrm{H}, 2 \mathrm{~d}, J=14 \mathrm{~Hz}, \mathrm{POCH}_{3}\right), 3.9-4.5\left[4 \mathrm{H}, \mathrm{m},\left(\mathrm{CH}_{2} \mathrm{O}\right)_{2}\right], 4.66,5.01(1 \mathrm{H}$, 
2s, CH). MS : 264 (M+, 16), 136 (81), 107 (100).

5- $\alpha$-Chlorobenzyl-2-methoxy-5-propyl-1, 3, 2-dioxaphosphorinane 2-Sulfide (MP18)

'H-NMR: $0.92\left[3 \mathrm{H}, \mathrm{t}, J=7 \mathrm{~Hz}, \mathrm{CH}_{3}(\mathrm{Pr})\right], 1.1-1.9\left(4 \mathrm{H}, \mathrm{m}, \mathrm{MeCH}_{2} \mathrm{CH}_{2}\right), 3.79,3.84$ $\left(3 \mathrm{H}, 2 \mathrm{~d}, J=14 \mathrm{~Hz}, \mathrm{POCH}_{3}\right), 4.0-4.8\left[4 \mathrm{H}, \mathrm{m},\left(\mathrm{CH}_{2} \mathrm{O}\right)_{2}\right], 5.22,5.64(1 \mathrm{H}, 2 \mathrm{~s}, \mathrm{PhCH}), 7.32$ (5H, s, Ph). MS : $334\left(\mathrm{M}^{+}, 7\right), 209$ (41), 171 (42), 129 (100).

2-Methoxy-5-(2-phenylvinyl)-5-propyl-1, 3, 2-dioxaphosphorinane 2-Sulfide (MP19)

${ }^{1} \mathrm{H}-\mathrm{NMR}$ : $0.94\left(3 \mathrm{H}, \mathrm{t}, J=7 \mathrm{~Hz}, \mathrm{CCH}_{3}\right), 1.1-1.8\left(4 \mathrm{H}, \mathrm{m}, \mathrm{MeCH}_{2} \mathrm{CH}_{2}\right), 3.76,3.79(3 \mathrm{H}$, $2 \mathrm{~d}, J=14 \mathrm{~Hz}, \mathrm{POCH},), 4.0-4.6\left[4 \mathrm{H}, \mathrm{m},\left(\mathrm{CH}_{2} \mathrm{O}\right)_{2}\right], 5.7-6.7(2 \mathrm{H}, \mathrm{m}, \mathrm{CH}=\mathrm{CH}), 7.28(5 \mathrm{H}, \mathrm{m}$, $\mathrm{Ph})$. MS : $312(\mathrm{M}+, 100)$.

\section{Transformation of M P-1 and MP-5 into BP-I and BP-Z}

The reaction was carried out under the conditions shown in Table 1 , monitored by ${ }^{31} \mathrm{P}-\mathrm{NMR}$ (Fig. 2). The reaction mixture in the appropriate solvent $(1.5 \mathrm{ml})$ was put in an $8 \mathrm{~mm} \phi \mathrm{NMR}$ tube and the tube was inserted into a $10 \mathrm{~mm} \phi$ NMR tube containing $0.5 \mathrm{ml} \mathrm{CDCl}_{3}$ as a locking solvent to measure ${ }^{31} \mathrm{P}-\mathrm{NMR}$. The reaction products were identified by comparing the chemical shift values with those of reference compounds (MP-1, 5 and BP-1, 2).

\section{Chromatographies, spectroscopies and other method}

Thin-layer silica gel plates $(60 \mathrm{~F}-254,0.25 \mathrm{~mm}$ thick ; Merck) were utilized for analytical thin-layer chromatography (TLC).

Column chromatography was performed on Merck silica gel 60 of particle size 0.063-0.2 mm.

${ }^{1} \mathrm{H}$ - and ${ }^{31} \mathrm{P}-\mathrm{NMR}$ were measured with a JEOL JNM-FX 100 spectrometer at 100 $\mathrm{MHz}$ and $40 \mathrm{MHz}$, respectively. Tetramethylsilane (TMS) was used as an internal standard for ${ }^{1} \mathrm{H}$-NMR and $85 \%$ phosphoric acid as an external standard for ${ }^{31} \mathrm{P}-\mathrm{NMR}$ measurements. Chemical shift values were expressed in $\delta\left(\mathrm{CDCl}_{3}\right) \mathrm{ppm}$.

Mass spectra (MS) were obtained using two sets of mass spectrometer : an ESCO05A equipped with an ESCO MD-DP05G data processing system and a JEOL JMSD300 equipped with a JEOL JMA 3500 data processing system, ionizing voltage being at $70 \mathrm{eV}$ and $30 \mathrm{eV}$, respectively. Ion peaks were expressed in $m / z$, followed by the percentage of intensity in parentheses.

All melting points were determined on an MRK (Mitamura Riken Kogyo) apparatus and are uncorrected.

\section{M ethods of bioassay}

Three- to five-day old houseflies (Musca domestica Linne, WHO's standard susceptible strain, SRS) were used. Ten houseflies were topically treated with an acetone solution of a test sample. The mortality was counted $24 \mathrm{hr}$ after treatment. Experiments were dupricated. 


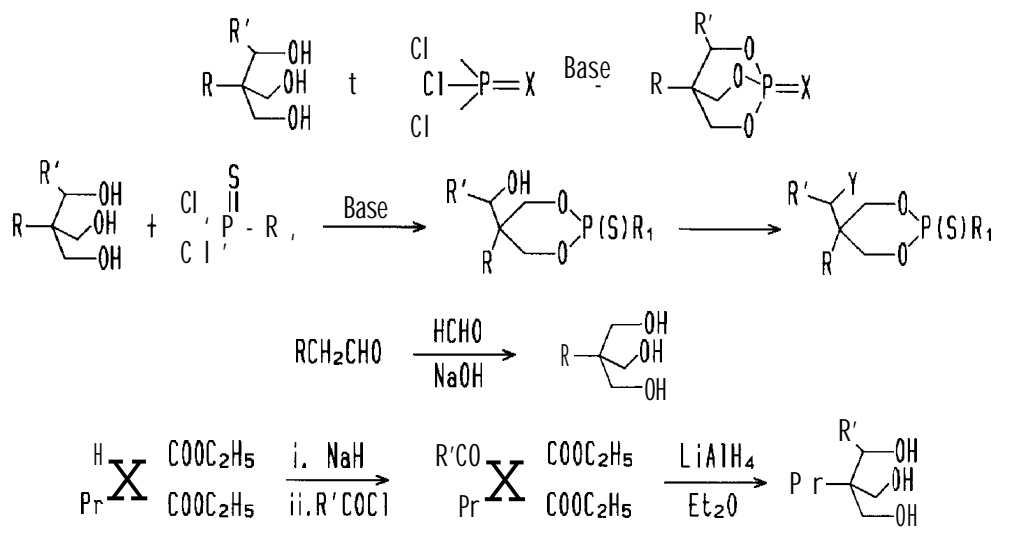

Fig. 1. Synthetic routes for bicyclic phosphorus esters (BPs) and related monocyclic phosphorothionates (MPs). $\mathrm{X}=0, \mathrm{~S}, \mathrm{Y}=\mathrm{OH}, \mathrm{OAc}, \mathrm{OMs}, \mathrm{OTs}, \mathrm{Br}, \mathrm{Cl}$.

Table 1. Transformation of MP-1 into BP-1 $(B)$ and BP-2 (A).

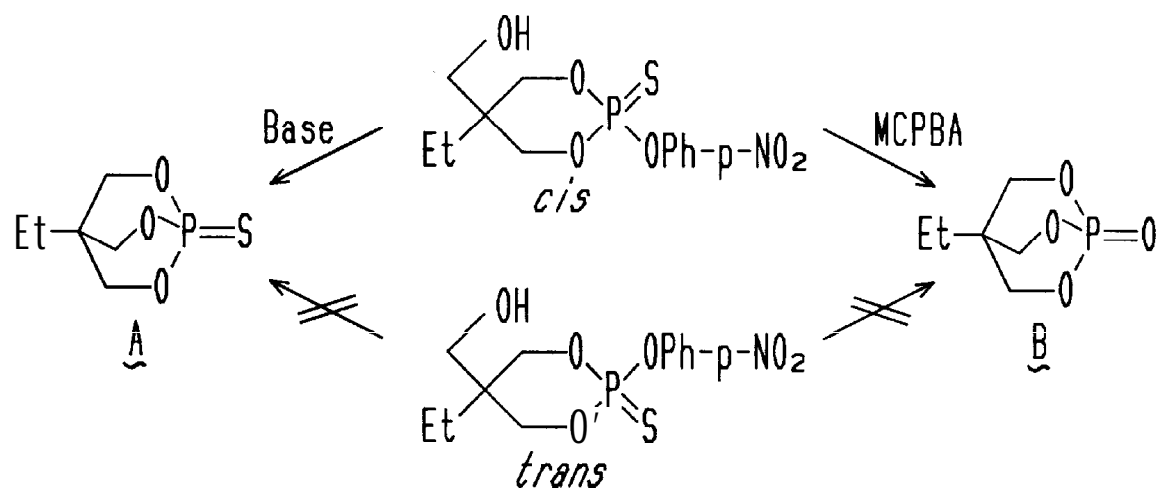

\begin{tabular}{|c|c|c|c|}
\hline \multirow{2}{*}{ Diastereomers } & \multirow{2}{*}{ Reaction conditions } & \multicolumn{2}{|c|}{ Yield (\%) } \\
\hline & & A & $B$ \\
\hline cis & benzene, reflux, $2 \mathrm{hr}$ & 0 & 0 \\
\hline cis & benzene, pyridine (2equiv.), r. t. $2 \mathrm{hr}$ & 21 & 0 \\
\hline cis & acetone, pyridine (2equiv.), r. t. $2 \mathrm{hr}$ & 14 & 0 \\
\hline cis & acetone, $\mathrm{K}_{2} \mathrm{CO}_{3}$ excess, r. t. $15 \mathrm{~min}$ & 10 & $\mathrm{c}$ \\
\hline cis & acetone, $\mathrm{K}_{2} \mathrm{CO}_{3}$ excess, reflux, $\mathrm{hr}$ & 100 & 0 \\
\hline trans & acetone, $\mathrm{K}_{2} \mathrm{CO}_{3}$ excess, reflux, $\mathrm{lhr}$ & 0 & 0 \\
\hline cis & $\mathrm{CHCl}_{3}, \mathrm{MCPBA}$ (equiv.), r. t. $10 \mathrm{~min}$ & 0 & 33 \\
\hline cis & $\mathrm{CHCl}_{3}, \mathrm{MCPBA}$ (2equiv.), r. t. $30 \mathrm{~min}$ & 0 & 95 \\
\hline trans & $\mathrm{CHCl}_{3}, \mathrm{MCPBA}$ (2equiv.), r. t. $30 \mathrm{~min}$ & 0 & 0 \\
\hline
\end{tabular}

\section{RESULTS AND DISCUSSIONS}

\section{Chemistry}

Fig. 1 summarizes the synthetic schemes of BPs and MPs. The triols having different $R$ and $\mathbf{R}^{\prime}$ groups were synthesized from the corresponding aldehydes or 
Table 2. Insecticidal activity of BP against susceptible housefly.

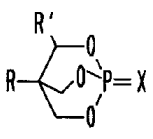

\begin{tabular}{|c|c|c|c|c|c|c|}
\hline \multirow{2}{*}{ Compd. } & \multirow{2}{*}{ No. } & \multirow{2}{*}{$\mathrm{X}$} & \multirow{2}{*}{$\mathrm{R}$} & \multirow{2}{*}{$\mathrm{R}^{\prime}$} & \multicolumn{2}{|c|}{$L_{50}(\mu \mathrm{g} / \mathrm{fly})$} \\
\hline & & & & & alone & $+\mathrm{PB}(5 \mu \mathrm{g})$ \\
\hline BP- 1 & & $\mathrm{O}$ & $\mathrm{Et}$ & $\mathrm{H}$ & $>50$ & $\mathrm{NT}^{*}$ \\
\hline BP- 2 & & $\mathrm{~s}$ & $\mathrm{Et}$ & $\mathrm{H}$ & 14.42 & NT \\
\hline BP- 3 & & $\mathrm{~S}$ & $\mathrm{Ph}$ & $\mathrm{H}$ & 4.98 & NT \\
\hline BP- 4 & & $\mathrm{~S}$ & c-Hex & $\mathrm{H}$ & 1.76 & NT \\
\hline BP- 5 & & $\mathrm{~S}$ & $\mathrm{Pr}$ & $\mathrm{H}$ & 0.22 & 0.12 \\
\hline BP- 6 & & $\mathrm{~S}$ & $\operatorname{Pr}$ & $\mathrm{Me}$ & 0.24 & 0.13 \\
\hline BP- 7 & & $\mathrm{~S}$ & $\operatorname{Pr}$ & $\mathrm{Et}$ & 0.58 & 0.16 \\
\hline BP- 8 & & $\mathrm{~S}$ & $\operatorname{Pr}$ & i-Pr & 0.75 & 0.11 \\
\hline BP- 9 & . & $\mathrm{S}$ & $\operatorname{Pr}$ & $\mathrm{Ph}$ & $>100$ & NT \\
\hline BP-10 & & $\mathrm{S}$ & $\operatorname{Pr}$ & $\mathrm{Bz}$ & $>100$ & NT \\
\hline
\end{tabular}

*not tested.

Table 3. Insecticidal activity of MP against susceptible housefly.

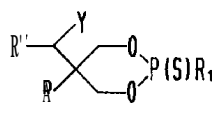

\begin{tabular}{|c|c|c|c|c|c|c|}
\hline \multirow{2}{*}{ Compd. No. } & \multirow{2}{*}{$\mathrm{R}$} & \multirow{2}{*}{$\mathrm{R}^{\prime}$} & \multirow{2}{*}{$\mathrm{R}_{1}$} & \multirow{2}{*}{$\mathrm{Y}$} & \multicolumn{2}{|c|}{$\mathrm{LD}_{50}(\mu \mathrm{g} / \mathrm{fly})$} \\
\hline & & & & & cis & trans \\
\hline MP- 1 & Et & $\mathrm{H}$ & $\mathrm{O} \cdot p \cdot \mathrm{NO}_{2} \cdot \mathrm{Ph}$ & $\mathrm{OH}$ & \multicolumn{2}{|c|}{$>50$} \\
\hline MP- 2 & Et & $\mathrm{H}$ & $\mathrm{O} \cdot p \cdot \mathrm{NO}_{2} \cdot \mathrm{Ph}$ & $\mathrm{OAc}$ & \multicolumn{2}{|c|}{$>50$} \\
\hline MP- 3 & Et & $\mathrm{H}$ & $\mathrm{OMe}$ & $\mathrm{OH}$ & \multicolumn{2}{|c|}{$>100$} \\
\hline MP- 4 & Et & $\mathrm{H}$ & $\mathrm{OMe}$ & $\mathrm{OAc}$ & \multicolumn{2}{|c|}{$>100$} \\
\hline MP- 5 & Et & $\mathrm{H}$ & $\mathrm{SMe}$ & $\mathrm{OH}$ & \multicolumn{2}{|c|}{$>100$} \\
\hline MP- 6 & Et & $\mathrm{H}$ & $\mathrm{SMe}$ & $\mathrm{OAc}$ & \multicolumn{2}{|c|}{$>100$} \\
\hline MP- 7 & Et & $\mathrm{H}$ & SEt & $\mathrm{OH}$ & \multicolumn{2}{|c|}{$>100$} \\
\hline MP- 8 & Et & $\mathrm{H}$ & $\mathrm{OMe}$ & $\mathrm{OMs}^{\mathrm{a}}$ & \multicolumn{2}{|c|}{$>100$} \\
\hline MP- 9 & Et & $\mathrm{H}$ & $\mathrm{OMe}$ & $\mathrm{OTs}^{\mathrm{b}}$ & \multicolumn{2}{|c|}{$>100$} \\
\hline MP-10 & Et & $\mathrm{H}$ & OMe & $\mathrm{Br}$ & \multicolumn{2}{|c|}{$>50$} \\
\hline MP-11 & Et & $\mathrm{H}$ & $\mathrm{OMe}$ & $\mathrm{Cl}$ & \multirow[t]{2}{*}{$>100$} & 27.15 \\
\hline MP-12 & Et & $\mathrm{H}$ & $\mathrm{Cl}$ & $\mathrm{OH}$ & & - \\
\hline MP-13 & Et & $\mathrm{H}$ & $\mathrm{Cl}$ & $\mathrm{Cl}$ & $>11065$ & 3.96 \\
\hline MP-14 & $\mathrm{Pr}$ & $\mathrm{H}$ & $\mathrm{OMe}$ & $\mathrm{Cl}$ & 15.19 & 10.32 \\
\hline MP-15 & $\operatorname{Pr}$ & $\mathrm{Me}$ & $\mathrm{OMe}$ & $\mathrm{Cl}$ & 10.29 & 7.73 \\
\hline MP-16 & $\mathrm{Pr}$ & $\mathrm{Et}$ & $\mathrm{OMe}$ & $\mathrm{Cl}$ & \multicolumn{2}{|c|}{56.57} \\
\hline MP-17" & $\operatorname{Pr}$ & $\mathrm{Me}$ & OMe & $\mathrm{Me}$ & \multicolumn{2}{|c|}{$>100$} \\
\hline MP-18 & $\operatorname{Pr}$ & $\mathrm{Ph}$ & $\mathrm{OMe}$ & $\mathrm{Cl}$ & \multicolumn{2}{|c|}{$>100$} \\
\hline MP-19' & $\operatorname{Pr}$ & $\mathrm{Ph}$ & OMe & $\mathrm{H}$ & \multicolumn{2}{|c|}{$>100$} \\
\hline
\end{tabular}

a Ms ; methanesulfonyl.

b Ts ; $p$-toluenesulfonyl.

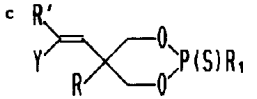




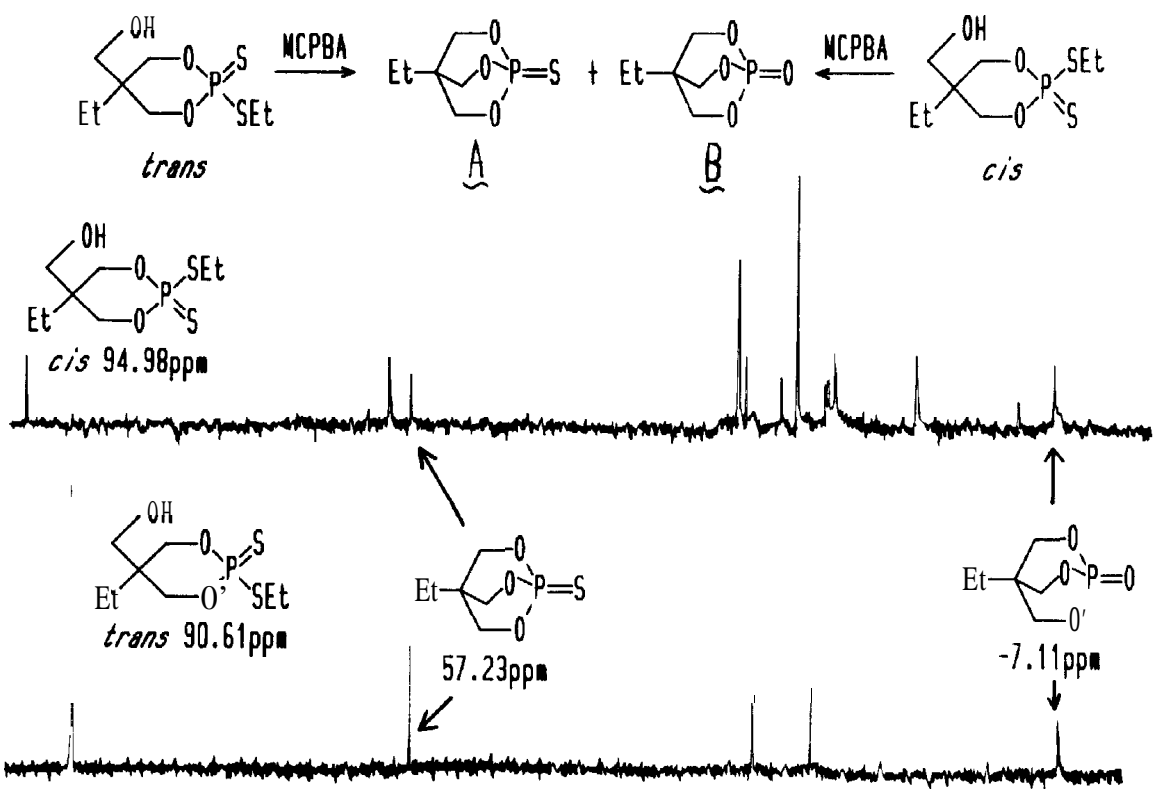

Fig. 2. The transformation of cis- and trans-MP-7 into BP-1 (B) and BP-2 (A) with MCPBA monitored by ${ }^{31} \mathrm{P}-\mathrm{NMR}$.

diethyl malonates (Ozoe and Eto, 1982).

The structures of some MPs were designed as a monocyclic phosphorothionate having a nucleophilic group, which was either protected or non-protected by a biodegradable group, at position 5 and a leaving group at position 2. In addition, some MPs with a chloro-functionized 5-substituent were synthesized with expectations to form thiolate type BPs via intra-molecular S-alkylation (Hirashima and Eto, 1983). Table 1 illustrates that the cis-p-nitrophenyl ester MP-1 was transformed into BP-1 (B) or BP-2 (A), by MCPBA oxidation or with action of a base, respectively. With trans-MP1 as a starting material, such transformations were not observed. However, both cisand trans-dithioate MP-7 gave a mixture of BP-1 (B) and BP-2 (A) by MCPBA oxidation (Fig. 2). These transformations could also be expected to occur in vivo because similar oxidation and cyclization are known to occur in organism (Eto et al., 1967; Eto, 1974).

\section{Insecticidal activity}

Table 2 shows the insecticidal activity of the prepared BPs. Oxo-type BPs were not toxic to insects by topical application unless the insect was pretreated with $\mathrm{PB}$ (Ozoe et al., 1983). An alternation of $\mathrm{P}=\mathrm{O}$ to $\mathrm{P}=\mathrm{S}$ improved the insecticidal activity, supporting the finding of Ozoe et al. (1986b). n-Propyl group was most favorable as a substituent at position 4 for the insecticidal activity. In the case of oxo-type BPs, the introduction of methyl group at position 3 increased the insecticidal activity against houseflies by injection and decreased mammalian toxicity (Ozoe et $a l$., 1983). In the case of thiono-type BPs, however, the introduction of an alkyl group at position 3 did not improve the topical insecticidal activity. The difference of insecticidal activity 
between a 3-hydrogen derivative (BP-5) and 3-alkyl derivatives (BP-6, 7 and 8) might relate to the oxidative detoxication, because $\mathrm{PB}$, a synergist which inhibits the oxidative metabolism (Eto, 1974), increased the insecticidal activity of BP-5, 6, 7 and 8 to a same level. Introduction of a phenyl group (BP-9) or a benzyl group (BP-10) at position 3 decreased the activity dramatically, compared with BP-5, 6, 7 and 8 .

On the other hand, most of MPs (MP-1-9 and 16 19), examined as the diastereomeric mixtures, showed no insecticidal activity against houseflies at a dose of 50 or $100 \mu \mathrm{g} /$ fly by topical application (Table 3), as opposed to the expectations to cyclize to the corresponding BP (Table 1 and Fig. 2). The difference of insecticidal activity between the cis- and trans-isomers of some MPs having a chloro-functionized 5substituent (MP-11 and 13-15) was not large. Trans-MP-13, 14 and 15 showed a little higher insecticidal activity than the corresponding c\&-isomers. Trans-MP-11 was much more active than cis-MP-11. However, their cyclization to thiolate type BPs was not proved yet. The insecticidal mode of action of the MPs having a chlorofunctionized 5-substituent is not known.

\section{ACKNOWLEDGEMENT}

This work was supported in part by the research grant from the Ministry of Education, Science, and Culture of Japan.

\section{REFERENCES}

Bellet, E. M. and J. E. Casida 1973 Bicyclic phosphorus esters. Science, 182 : 11351136

Bowery, N. G., J. F. Collins and R. G. Hill 1976 Bicyclic phosphorus esters that are potent convulsants and GABA antagonists. Nature, 261: 601-603

Eto, M. 1974 Organophosphorus Pesticides : Organic and Biological Chemist?. CRC Press, Cleaveland

Eto, M., Y. Oshima and J. E. Casida 1967 Plasma albumin as a catalyst in cyclization of diary1 o$(\alpha$-hydroxy)tolyl phosphates. Biochem. Pharmacol., $16: 295-308$

Eto, M., Y. Ozoe, T. Fujita and J. E. Casida 1976 Significance of branched bridge-head substituent in toxicity of bicyclic phosphorus esters. Agric.Biol.Chem., $40:$ 2113-2115

Fukuto, T. R. 1984 Propesticides. In "Pesticide Synthesis through Rational Approaches", ed. by P. S. Magee, G. K. Kohn and J. J. Menn, ACS Symposium Series 255, American Chemical Society, Washington, D. C., pp. 87-101

Hirashima, A. and M. Eto 1983 Stereochemistry and reactions of aziridinylphosphinothionates derived from amino acids. Agric. Biol.Chem., 47 : 829-838

Korenaga, S., Y. Ito, Y. Ozoe and M. Eto 1977 The effects of bicyclic phosphate esters on the invertebrate and vertebrate neuro-muscular junctions. Comp. Biochem. Physiol., 57C: $95-100$

Ozoe, Y. and M. Eto 1982 Synthesis and some spectral characteristics of bicyclic phosphate GABA antagonists. Agric. Biol. Chem., $46: 411-418$

Ozoe, Y., M. Eto, K. Mochida and T. Nakamura 1986a Characterization of high affinity binding of $\left[{ }^{3} \mathrm{H}\right]$ propyl bicyclic phosphate to house fly head extracts. Pestic. Biochem. Physiol., 26 : 263-274

O zoe, Y., H. Karino, K. Mochida, T. Nakamura and M. Fujii 1986b Penetration and metabolism of bicyclic phosphorus esters which affect their insecticidal activity against the housefly. $J$. Pesticide sci., 11: 433-439

Ozoe, Y., K. Mochida, T. Nakamura, A. Shimizu and M. Eto 1983 Toxicity of bicyclic phosphate GABA antagonists to the housefly, Musca domestica L. J. Pesticide Sci., 8: 601-605

Squires, R. F., J. E. Casida, M. Richardson and E. Saederup $1983\left[{ }^{35} \mathrm{~S}\right] t$-butylbicyclophosphoro- 
thionate binds with high affinity to brain-specific sites coupled to y-aminobutyric acid-A and ion recognition sites. Mol. Pharmacol., 23 : 326-336

Toia, R. F. and J. E. Casida 1985 Probicyclophosphates: Monocyclophosphates as potential prodrugs for bicyclophosphate GABA antagonists. Toxicol.Appl.Pharmacol., 81: 50-57

Toia, R. F. and J. E. Casida 1987 Probicyclophosphates as novel GABA antagonists. In "Biophosphates and Their Analogues-Synthesis, Structure, Metabolism and Activity,” ed. by K. S. Bruzik and W. J. Stec., Elsevier Science Publishers B. V., Amsterdam, The Netherlands, pp. 465-468 